\title{
SKLB-M8 Induces Apoptosis Through the AKT/mTOR Signaling Pathway in Melanoma Models and Inhibits Angiogenesis With Decrease of ERK1/2 Phosphorylation
}

\author{
Jingjing Wang ${ }^{1, \dagger}$, Zhuang Yang ${ }^{1,2, \dagger}$, Jiaolin Wen ${ }^{1}$, Feng Ma ${ }^{3}$, Fang Wang ${ }^{1}$, Kun $\mathrm{Yu}^{1}$, Minghai Tang ${ }^{1}$, \\ Wenshuang Wu ${ }^{1}$, Yinfeng Dong ${ }^{1}$, Xia Cheng ${ }^{1}$, Chunlai Nie ${ }^{1, * a}$, and Lijuan Chen ${ }^{1, * b}$ \\ ${ }^{1}$ State Key Laboratory of Biotherapy, West China Hospital, West China Medical School, Sichuan University, \\ Chengdu 610041, China \\ ${ }^{2}$ College of Chemistry, Sichuan University, Chengdu 610064, China \\ ${ }^{3}$ Institute of Blood Transfusion, Chinese Academy of Medical Sciences \& Peking Union Medical College, \\ Chengdu 610081, China
}

Received March 30, 2014; Accepted August 7, 2014

\begin{abstract}
SKLB-M8, a derivative of millepachine, showed significant anti-proliferative effects in melanoma cell lines. In this study, we investigated the anti-melanoma and anti-angiogenic activity of SKLB-M8 on three melanoma cell lines (A2058, CHL-1, and B16F10) and human umbilical vein endothelial cells (HUVECs). In vitro, SKLB-M8 showed anti-proliferative activity with $\mathrm{IC}_{50}$ values of $0.07,0.25$, and $0.88 \mu \mathrm{M}$ in $\mathrm{A} 2058$, CHL-1, and B16F10 cell lines, respectively. Flow cytometory analysis showed that SKLB-M8 induced G2/M arrest in three melanoma cell lines, and western blotting demonstrated that SKLB-M8 down-regulated the expression of cdc2, up-regulated p53 in A2058 and CHL-1 cells, and triggered cell apoptosis through down-regulating AKT and phosphorylated mTOR (p-mTOR). SKLB-M8 also inhibited HUVEC proliferation, migration, invasion, and tube formation in vitro with the inhibition of phosphorylated ERK1/2 (p-ERK1/2). In vivo, alginate-encapsulated tumor cell assay revealed that SKLB-M8 suppressed B16F10 tumor angiogenesis. In CHL-1- and B16F10-tumor-bearing mouse models, SKLB-M8 inhibited tumor growth by oral treatment with less toxicity. CD31 immunofluoresence staining and caspase-3 immunohistochemistry indicated that SKLB-M8 inhibited melanoma tumor growth by targeting angiogenesis and inducing caspase3-dependent apoptosis. SKLB-M8 might be a potential anti-melanoma drug candidate.
\end{abstract}

[Supplementary Figure: available only at http://dx.doi.org/10.1254/jphs.14077FP]

Keywords: melanoma, anti-angiogenesis, p53, AKT, ERK

\section{Introduction}

Malignant melanoma, one of the most aggressive and fatal categories of cancer, causes the majority of deaths related to skin cancer (1). However, no agents have shown statistically significant improvement with a good prognosis in the treatment of malignant melanoma. Discovery of more effective drugs with less toxicity are

${ }^{\dagger}$ These authors contributed equally to this work.

Corresponding authors.*aniec11022@scu.edu.cn,

*bchenlijuan125@163.com

Published online in J-STAGE on October 21, 2014

doi: $10.1254 /$ jphs.14077FP urgently needed. As there is an enormous structural diversity of biological sources, the compounds from natural products play an extremely important role in drug discovery and development of therapeutics. It is reported that almost half of the drugs introduced between 1940 and 2006 were closely related to natural products, especially in the area of cancer $(2-4)$, such as paclitaxel, a famous antitumor agent isolated from plants with good therapic effects.

Angiogenesis is essential for cancer cells to develop a blood center to supply enough oxygen and nutrients, thus ensuring tumor growth. Discovery of angiogenesis inhibitors has been a hot spot over the last couple of 
decades. Inhibiting endothelial cell growth (e.g., endostatin), blocking angiogenesis signaling (e.g. avastin), and extracellular matrix breakdown (e.g., inhibitors of MMPs) are three main targets of anti-angiogenic agents (5). Inducing cancer cells apoptosis are an extremely important therapeutics target in cancer therapy. P53 protein affects cell cycle progression and apoptosis (6), relating to the expression of cyclin B and cdc2 in G2 checkpoint control $(7,8)$. The mammalian target of rapamycin (mTOR), as an apoptosis inhibitor, could be regulated by PI3K/AKT, which plays a central role in tumor genesis and can activate a number of diverse downstream anti-apoptotic pathways $(9,10)$. Thus, we suggested that novel agents from natural products, which have anti-angiogenic effects and can induce cancer apoptosis, may be promising in melanoma therapy.

Millepachine (MIL), a novel chalcone with a 2,2dimethylbenzopyran motif, was first isolated from Millettia pachycarpa Benth (Leguminosae) by our group, which is a flavonoid-rich traditional Chinese medicine, has been used as a blood tonic in antihelminthic and cancer therapy for many years in China. In our previous studies, MIL was reported to be a potential lead compound for antitumor drug development and induced cell cycle arrest and apoptosis in human hepatocarcinoma cells (11). However, the poor solubility of MIL limited its further higher dose treatment. To improve the poor solubility and antitumor activity, MIL was further modified. Among a series of derivatives, $(E)$-3-(3-amino-
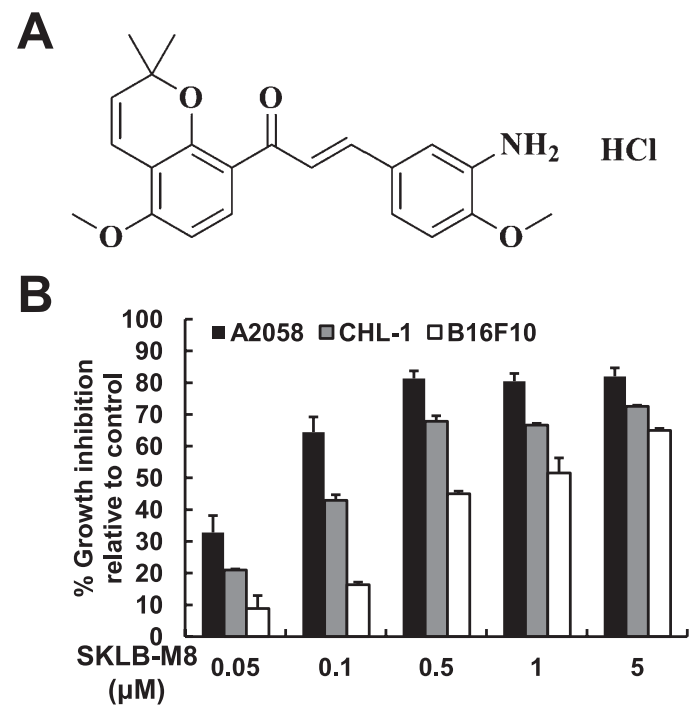

Fig. 1. SKLB-M 8 inhibited proliferation in melanoma cells. A: Structure of SKLB-M8. B: The inhibitory effect of SKLB-M8 on melanoma cell proliferation. The cells were treated with the indicated concentrations of SKLB-M8 for $48 \mathrm{~h}$. Columns, means; bars, S.D. $(\mathrm{n}=3)$.
4-methoxyphenyl)-1-(5-methoxy-2,2-dimethyl-2Hchromen-8-yl)prop-2-en-1-one hydrochloride (SKLBM8) (Fig. 1A) showed the most promising antitumor activity. Our studies detected that SKLB-M8 inhibited tubulin polymerization binding to the colchicine site, induced apoptosis with cell cycle arrest in HepG2 cells and cause rapid endothelial cell shape changes (data not shown). However, the antitumor activity in melanoma and the mechanisms of anti-angiogenic activity of SKLB-M8 were unknown.

In this study, SKLB-M8 exhibited strong antiproliferative activity in melanoma cell lines and the inhibitory effect of melanoma tumor growth was statistically significant in two mouse models (B16F10 and CHL-1). We discussed mechanisms of the anti-proliferative and apoptosis-inducing effects of SKLB-M8 and evaluated the anti-angiogenesis activity.

\section{Materials and Methods}

\section{Ethics statement}

Animal studies were conducted in conformity with the institutional guide for the care and use of laboratory animals. All mouse protocols were approved by the Animal Care and Use Committee of Sichuan University (Chengdu, Sichuan, China).

\section{Reagents and chemicals}

Dimethyl sulfoxide (DMSO), 3-(4,5-dimethylthiazol2-yl)-2,5-diphenyltetrazolium bromide (MTT), propidium iodide (PI), and antibodies against $\beta$-actin and caspase-9 were purchased from Sigma (St. Louis, MO, USA). 4',6Diamidino-2-phenylindole (DAPI) was from Beyotime Company of China. Antibodies against caspase-3, cyclin B1, cdc2, p53, total AKT, total and phosphomTOR, total and phospho-VEGFR2, total and phospho-FAK, total and phospho-Src, and total and phospho-ERK1/2 were obtained from Cell Signaling Technology (Danvers, MA, USA). SKLB-M8 (95\% or higher purity) was first synthesized by our group, and a $10 \mathrm{mM}$ stock solution was prepared in DMSO and diluted in optimal assay buffer or culture medium.

\section{Cell lines and cultures}

The human melanoma cell lines CHL-1 and A2058 and the murine melanoma cell line B16F10 were obtained from American Type Culture Collection (ATCC, Manassas, VA, USA). Cells were all cultured in Dulbecco's modified Eagle's medium, supplemented with $10 \%$ fetal bovine serum, and maintained in a $5 \%$ $\mathrm{CO}_{2}$ incubator at $37^{\circ} \mathrm{C}$. Human umbilical vein endothelial cells (HUVECs) were isolated from human umbilical cord veins by a standard procedure as pre- 
viously described and cultured in EBM-2 medium with SingleQuots (Lonza) containing VEGF and other growth factors (12).

\section{Cell proliferation assay}

Cells $\left(2 \times 10^{3}-1 \times 10^{4}\right.$ cells per well $)$ were seeded in 96-well culture plates overnight for attachment, then treated with vehicle alone or various concentrations of SKLB-M8 for $24 \mathrm{~h}$ (HUVECs) or $48 \mathrm{~h}$ (melanoma cells), and measured using the MTT assay with a Spectramax M5 Microtiter Plate Luminometer (Molecular Devices) as previously described (11). Each assay performed in triplicate.

\section{Flow cytometry assay}

Cells were treated with vehicle alone or various concentrations of SKLB-M8 for $24 \mathrm{~h}$, stained with PI $(50 \mu \mathrm{g} / \mathrm{ml})$, and analyzed by a flow cytometer (FASC240). Data were analyzed using Modfit 2.8 software.

\section{Morphological analysis of nuclei}

Cells were plated and treated with vehicle alone or SKLB-M8 for $24 \mathrm{~h}$. After the cells were fixed with $4 \%$ paraformaldehyde, they were incubated with DAPI staining solution and washed with PBS. Then, images were obtained by inverted fluorescence microscopy (Olympus, Tokyo).

\section{Wound healing assay}

Monolayer HUVECs were wounded by scratching with pipette tips and washed with PBS. Fresh EGM-2 medium containing vehicle or different concentrations of SKLB-M8 was added to the scratched monolayers. Cells were washed with PBS after $18-20 \mathrm{~h}$, fixed with $100 \%$ methanol for $15 \mathrm{~min}$, and then stained with $0.1 \%$ crystal violet for $20 \mathrm{~min}$ and washed with PBS. Images were taken by a fluorescence microscope (Carl Zeiss Microimaging Inc.). The cells were quantified by manual counting and the percentage of migrated cells inhibited by SKLB-M8 was expressed on the basis of vehicle wells.

\section{Transwell invasion assay}

Invasion assay was done as described previously (13). Briefly, the filter of the Transwell plate (Millipore, Bedford, MA, USA) was coated with $50 \mu$ l Matrigel (BD Biosciences, Bedford, MA, USA). After matrigel polymerization, the bottom chambers were filled with EGM-2 medium containing various growth factors and the top chambers were seeded with EBM-2 medium (without growth factors) and HUVECs $\left(2 \times 10^{4}\right.$ cells per well). The top chamber contained vehicle or various concentrations of SKLB-M8. After $24 \mathrm{~h}$, non-migrated cells were scraped off, and migrated cells were fixed with $100 \%$ methanol and stained with $0.1 \%$ crystal violet. After washing with PBS, the cells were quantified by manual counting and photographed under a light microscope. The percentage of inhibition was expressed using untreated cells at $100 \%$.

\section{Tube formation assay}

The tube formation assay was conducted as described previously (14). HUVECs $\left(1.5 \times 10^{4}\right)$ suspended in EBM-2 medium were seeded in 96-well culture plates after polymerization of the Matrigel and treated with SKLB-M8 or vehicle. After $6 \mathrm{~h}$, cells were photographed with an inverted microscope (Carl Zeiss Microimaging, Inc.).

\section{Western blot assay}

In assays for examining the mechanisms of action of SKLB-M8 on the melanoma cell lines, cells were incubated with SKLB-M8 for $24 \mathrm{~h}$ or $48 \mathrm{~h}$. Expression levels or activation status of apoptosis-signaling proteins, apoptosis-associated proteins, and cell cycle-related proteins were detected in B16F10, CHL-1, and A2058 melanoma cells.

To determine the effects of SKLB-M8 on the VEGFR2-dependent signaling cascade, HUVECs were serum-starved overnight and incubated with SKLB-M8 for $2 \mathrm{~h}$ and stimulated by $\operatorname{VEGF}_{165}(50 \mathrm{ng} / \mathrm{ml})$ for $10 \mathrm{~min}$. Protein samples were then subjected to $8 \%-15 \%$ sodium dodecylsulfate-polyacrylamide gel electrophoresis and transferred onto the polyvinylidene difluoride membrane. The membrane was blocked in 5\% non-fat milk and then incubated with primary antibody and subsequently with appropriate horseradish peroxidase-conjugated secondary antibody. Proteins were visualized with enhanced chemiluminescence (Millipore).

\section{Alginate-encapsulated tumor cell assay}

An alginate-encapsulated assay was conducted as described (15). Briefly, alginate beads containing $5 \times 10^{4}$ B16F10 cells per bead were formed and implanted s.c. into both dorsal sides of the C57 mice. Then mice were orally gavaged with SKLB-M8 $(20 \mathrm{mg} / \mathrm{kg})$ or vehicle daily for 14 days. At the end of the experiment, $0.1 \mathrm{ml}$ of $2 \%$ fluorescein isothiocyanate (FITC)-dextran solution (Sigma) was injected into the lateral tail vein of mice. Alginate beads were removed and photographed within 20 min after being exposed surgically. The uptake of FITC-dextran was measured as described (15).

\section{In vivo tumor xenograft study}

Six-week-old female mice were obtained from Chinese Academy of Medical Science (Beijing, China). 
B16F10 cells $\left(5 \times 10^{5}\right.$ in $100 \mu$ l saline $)$ were injected subcutaneously into the right flanks of C57 mice, and CHL-1 cells $\left(5 \times 10^{6}\right.$ in $100 \mu \mathrm{l}$ saline $)$ were injected subcutaneously into the right flanks of female nude mice (BALB/cA-nu $[\mathrm{nu} / \mathrm{nu}]$ ). When the tumor reached about $100 \mathrm{~mm}^{3}$, mice were selected and randomly divided. The animals were orally gavaged SKLB-M8 $(20 \mathrm{mg} / \mathrm{kg})$ or vehicle daily. Tumor burden was measured every 2 or 3 days with a caliper [calculated volume $\left(\mathrm{mm}^{3}\right)=\pi / 6 \times$ length $\times$ width $\times$ width]. After treatment, mice were killed and some internal organs (heart, liver, spleen, lung, and kidney) and tumors were collected.

\section{Histological analyses}

To investigate the anti-angiogenesis effect of SKLBM8, detection of vessel density in tumor tissue was done as described previously with some changes (16). Briefly, frozen sections of B16F10 tumor xenografts in optimal cutting temperature (OCT) medium were fixed with $4 \%$ paraformaldehyde, washed with PBS, blocked with 5\% normal goat serum, and stained with anti-CD31 antibody (1:50; BOSTER, Wuhan, China) in $2.5 \%$ normal goat serum. After overnight incubation, sections were washed, treated with secondary goat anti-rat Cy3 antibody (Beyotime, Shanghai, China), and further treated with DAPI staining solution.

The excised tumors and tissues were fixed in $4 \%$ paraformaldehyde for more than $24 \mathrm{~h}$ and embedded in paraffin. Sections of the tissues $(3-5 \mu \mathrm{m})$ were stained with hematoxylin and eosin. The effect of SKLB-M8 on inducing apoptosis was evaluated by immunostaining with active caspase-3.

\section{Statistical analyses}

All data were expressed as the mean \pm standard deviation (S.D.) and statistically compared by one-way analysis of variance with Student's $t$-test in different experiments, and $P<0.05$ was taken as statistically significant.

\section{Results}

SKLB-M8 strongly inhibited proliferation of melanoma cells

In the pre-experiment of anticancer compounds screening, SKLB-M8 (Fig. 1A) showed strong antiproliferative activity on several cancer cell lines, including melanoma (data not shown). The growth inhibition profile was assessed by MTT assay on three melanoma cell lines (A2058, CHL-1, B16F10) with $\mathrm{IC}_{50}$ values of $0.07,0.25$, and $0.88 \mu \mathrm{M}$, respectively (Fig. $1 \mathrm{~B}$ ).
SKLB-M8 induced G2/M phase arrest in melanoma cells

To investigate the effects on cell cycle regulation, three melanoma cell lines treated with SKLB-M8 were detected by flow cytometry. Under the concentration of $0.5 \mu \mathrm{M}$ SKLB-M8, the percent of cells in the G2/M fraction significantly increased compared to the control on A2058 cells $(15.81 \% \pm 1.94 \%$ to $51.00 \% \pm 7.16 \%)$ accompanied by a decrease in G0/G1 cells $(50.10 \% \pm$ $2.55 \%$ to $1.00 \% \pm 0.16 \%$ ). Similar results were observed on CHL-1 and B16F10 cells (Fig. 2A). These experiments revealed that SKLB-M8 induced G2/M arrest in melanoma cells.

In order to further understand the mechanisms of action, the expression levels of a number of key proteins involved in the G2/M phase transition were measured. Maturation-promoting factor (MPF) complex, which is formed of cyclin B and cdc2 protein kinase (CDK1), is essential for transition from G2 to mitosis (17). The results of up-regulation of cyclin $\mathrm{B} 1$ and down-regulation of cdc2 (Fig. 2B) induced by SKLB-M8 directly disrupted formation of MPF, which revealed that A2058 and CHL- 1 cells treated with SKLB-M8 were arrested in the $\mathrm{G} 2 / \mathrm{M}$ phase. We found that p53 was also upregulated, which might contribute to the G2/M block through decreasing the level of cdc2 (Fig. 2B), as described in previous reports $(8,17)$.

\section{SKLB-M8 induced apoptosis in melanoma cells}

From the morphological staining of cells with DAPI, chromosomes haphazardly scattered in the cell nucleus could be seen clearly (Fig. 3A), indicating that SKLBM8 might induce apoptosis in the three melanoma cell lines (A2058, CHL-1, B16F10). As caspases play a central role in apoptosis (18), we examined the activity of caspase- 3 and caspase- 9 by western blotting. Figure $3 \mathrm{~B}$ showed that the level of cleaved caspase- 3 increased and that of procaspase- 9 decreased in the three types of melanoma cells treated with $0.5 \mu \mathrm{M}$ SKLB-M8 for $48 \mathrm{~h}$. Moreover, PARP, one of the caspase-3 substrates (19), was also detected with the reduction of pro-PARP and the appearance of the cleaved fragment at $0.5 \mu \mathrm{M}$ (Fig. 3B). The results indicated that SKLB-M8 induced apoptosis in the three types of melanoma cells with the activation of caspase-3, caspase-9, and PARP.

AKT mediates the cell survival signals by phosphorylation and inhibition of several proapoptotic proteins, including caspase-9 (20). As Fig. 3C shows, the expression level of total AKT was down-regulated after a 48-h exposure to SKLB-M8 at $0.5 \mu \mathrm{M}$. MTOR and its phosphorylated form, which can regulate AKT, were also investigated (21). We found that the expression of p-mTOR significantly reduced in $0.5 \mu \mathrm{M}$ SKLB-M8treated B16F10 cells. 


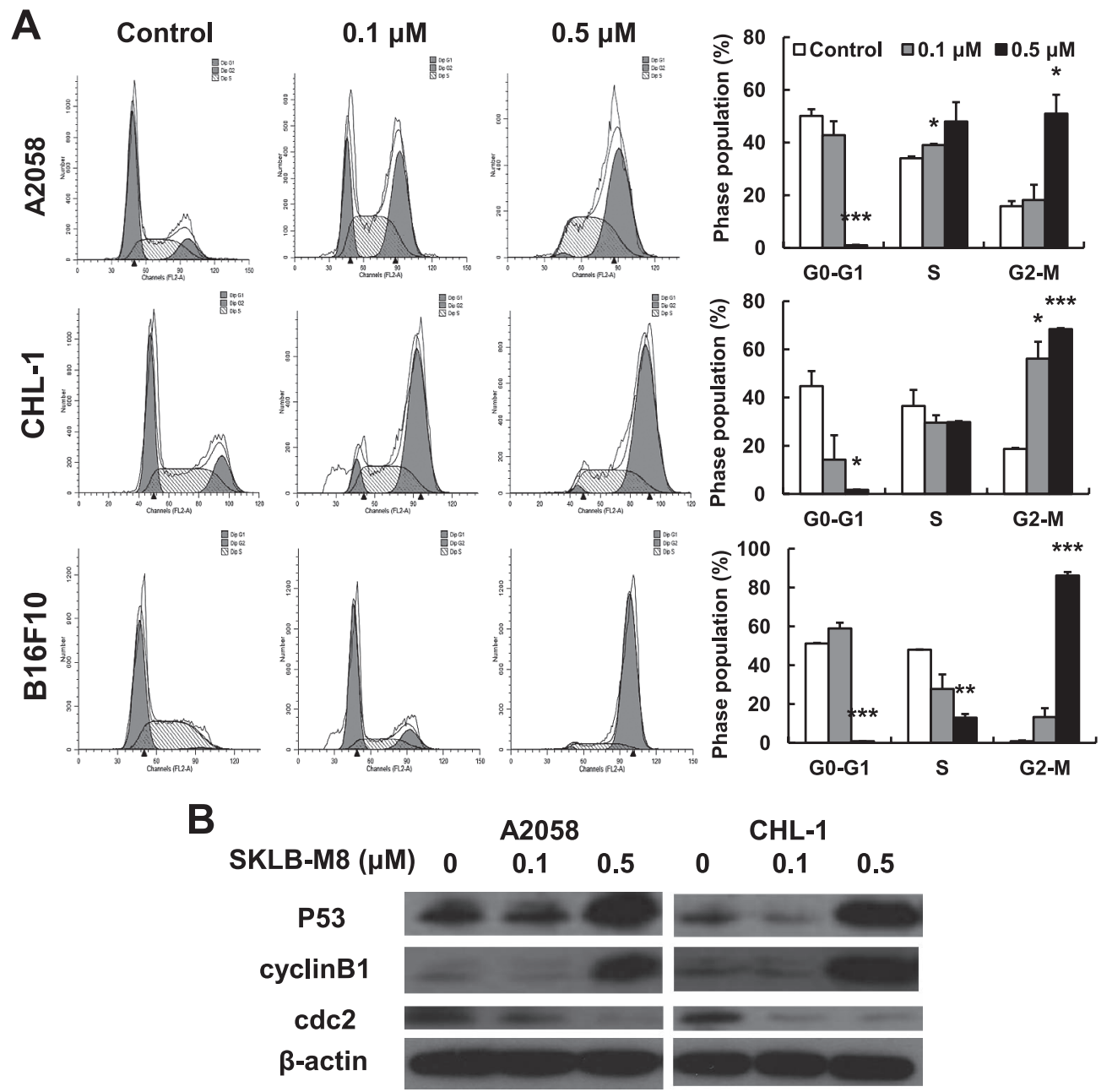

Fig. 2. SKLB-M8 induced G2/M phase arrest in melanoma cells. A: FCM analysis of cell cycle arrest in melanoma cells (A2058, CHL-1, and B16F10) with vehicle or SKLB-M8 treatment. SKLB-M8 caused G2/M cell cycle arrest. B: SKLB-M8 up-regulated cyclin B1 and p53 and down-regulated cdc2 in A2058 and CHL-1 cells.

SKLB-M8 inhibited HUVECs proliferation, migration, invasion, and tube formation with decrease of p-ERK1/2

Considering the inhibitory effect of SKLB-M8 on tubulin polymerization, which is related to angiogenic network maintenance and stabilization (22), we assessed the anti-angiogenic activity of SKLB-M8 in vitro. We first evaluated its inhibitory effects on VEGF-induced proliferation of HUVECs by MTT assay. SKLB-M8 significantly inhibited HUVECs proliferation with an $\mathrm{IC}_{50}$ value of $6.45 \mu \mathrm{M}$ after $24-\mathrm{h}$ treatment. It was known that angiogenesis demands migration and invasion of endothelial cells (14). Effects of SKLB-M8 on cell migration and invasion were evaluated. We found that $0.5 \mu \mathrm{M}$ SKLB-M8 obviously inhibited the migration of HUVECs with the inhibition rate of $81.25 \% \pm 2.83 \%$ in wound healing assays (Fig. 4A) and inhibited the invasion with inhibition rate of $75.87 \% \pm 10.61 \%$ in transwell assays (Fig. 4B). In tube formation assays, capillary-like structures were formed in the control group on the surface of matrigel within $6-8 \mathrm{~h}$, while treatment with SKLB-M8 apparently inhibited the process (Fig. 4C). We found that $0.1 \mu \mathrm{M}$ SKLB-M8 inhibited tube formation of HUVECs by $84.65 \% \pm 2.49 \%$, and $0.5 \mu \mathrm{M}$ almost completely blocked it. As shown in Fig. 4, SKLB-M8 has anti-angiogenic efficacy in HUVECs.

Previous studies reported that VEGFR2 and its downstream signaling are responsible for endothelial cell migration and tube formation (23). To verify the roles of the downstream effectors of VEGFR2 in angiogenesis, we investigated their expression in the presence of VEGF (Fig. 4D). The phosphorylation of VEGFR2, FAK, and Src induced by VEGF were not changed, but $5 \mu \mathrm{M}$ SKLB-M8 significantly suppressed p-ERK1/2, which suggested that SKLB-M8 mainly inhibited the 
A
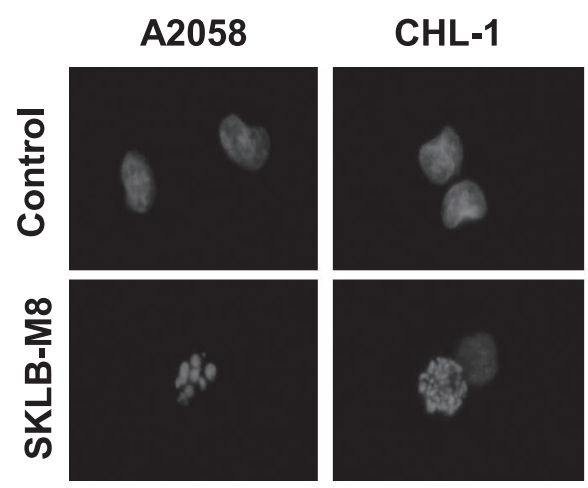

B

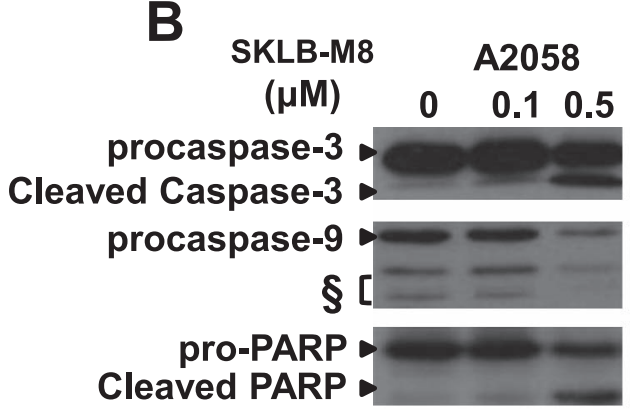

CHL-1
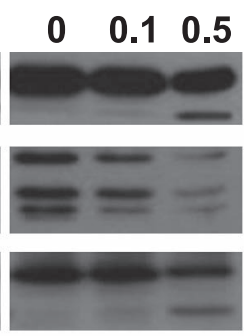

C
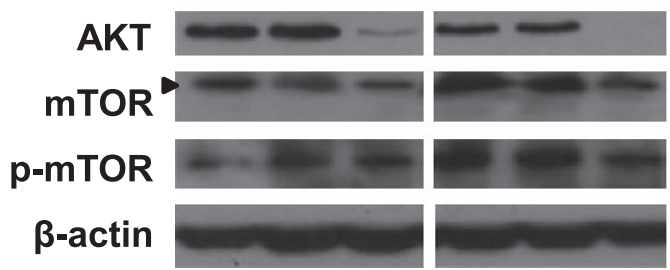

B16F10

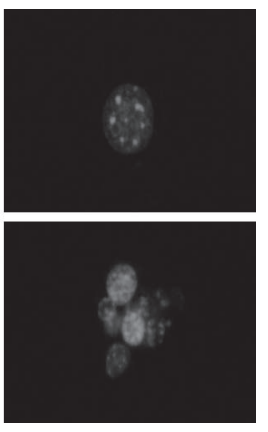

B16F10
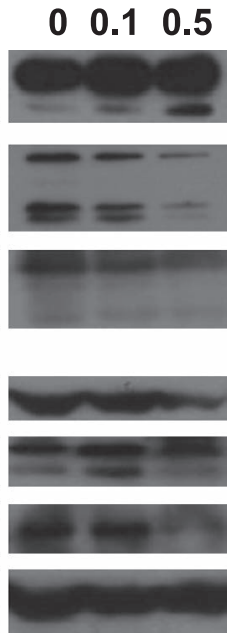

Fig. 3. SKLB-M8 induced apoptosis in melanoma cells. A2058, CHL-1, and B16F10 cells were treated with SKLB-M8 at various concentrations and assessed by immunostaining and western blotting. A: The morphological analysis of nuclei. B: SKLB-M8 induced apoptosis through activating caspase-3,9 and PARP. ( $§$, nonspecific bands). C: SKLB-M8 induced apoptosis through the AKT/ mTOR pathway.

activation of ERK1/2 to exert its anti-angiogenic function in endothelial cells.

\section{SKLB-M8 inhibited melanoma angiogenesis in vivo}

In order to better mimic the process of angiogenesis induced by tumor cells in vivo and determine the inhibitory effect of SKLB-M8 on it, we performed an alginate-encapsulate B16F10 cell assay as described previously $(15,24,25)$. A highly sensitive and relevant in vivo model was established to demonstrate the whole process of tumor angiogenesis and quantify the inhibitory action of anti-angiogenic compounds related to their antitumor activity (15). As Fig. 5 shows, new blood vessels in alginate beads from SKLB-M8-treated mice were apparently sparse and the FITC-dextran uptake was significantly decreased in comparison with control groups. These results suggested that SKLB-M8 could restrict tumor growth by inhibiting angiogenesis.

\section{SKLB-M8 inhibited melanoma growth in vivo}

We examined the in vivo anti-melanoma activity of SKLB-M8 using the B16F10 and CHL-1 models. In the
B16F10 tumor model, the mice were treated orally once daily with $20 \mathrm{mg} / \mathrm{kg}$ for 11 days. At the end of treatment, we found that $20 \mathrm{mg} / \mathrm{kg}$ SKLB-M8 once daily could suppress tumor growth with a tumor inhibition rate of about $58.22 \%$ compared with the control group (Fig. 6: A, C). In CHL-1 xegograft model, the inhibition rate of $20 \mathrm{mg} / \mathrm{kg}$ SKLB-M8 once daily was $61.41 \%$ after 21 days of treatment (Fig. 6: B, D).

Histological analysis was performed in the B16F10 model to verify SKLB-M8's anti-melanoma mechanisms of action. Immunofluorescence anti-CD31 staining of the B16F10 tumor tissue from SKLB-M8-treated mice showed significantly decreased microvessel density compared with control groups (Fig. 6E). Immunohistochemical analysis revealed that the percentage of active caspase-3-expressing cells in tumor tissue was significantly higher following SKLB-M8 treatment as we detected in vitro (Fig. 6F).

Furthermore, to evaluate the health status of mice treated with SKLB-M8, weights of the mice were monitored throughout the whole experiment. As shown in Supplementary Fig. 1A (available in the online version 

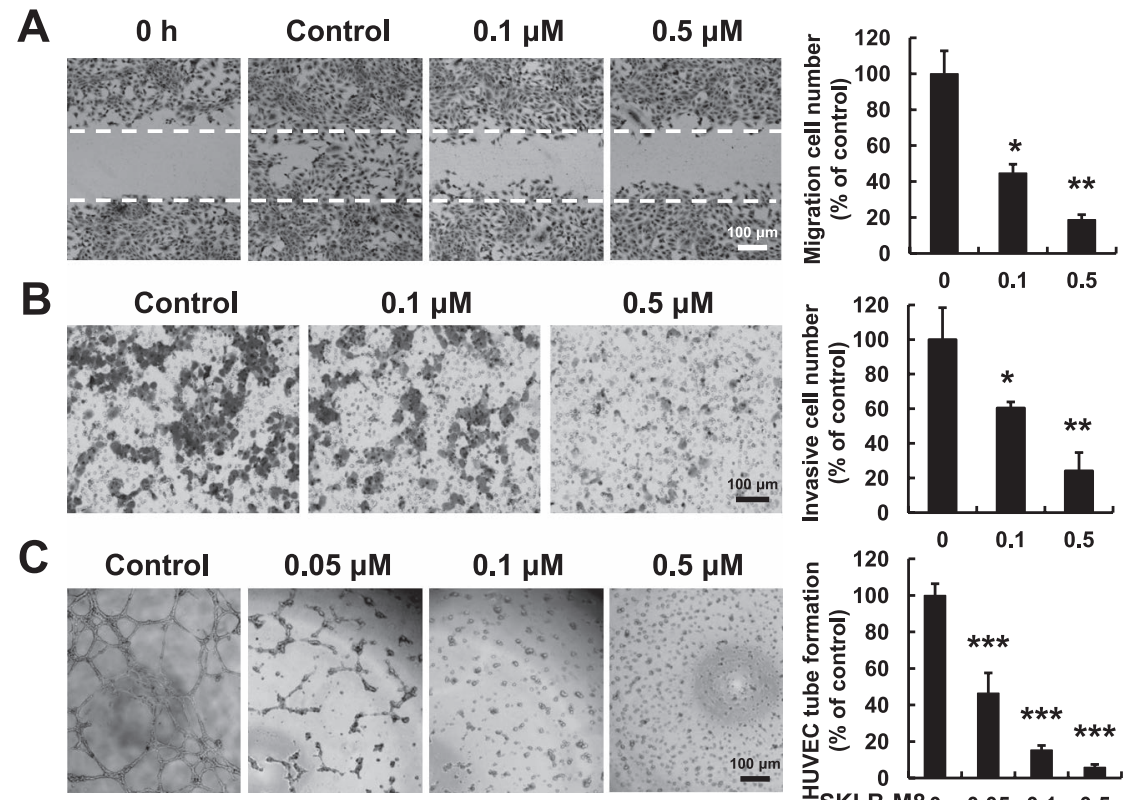

D

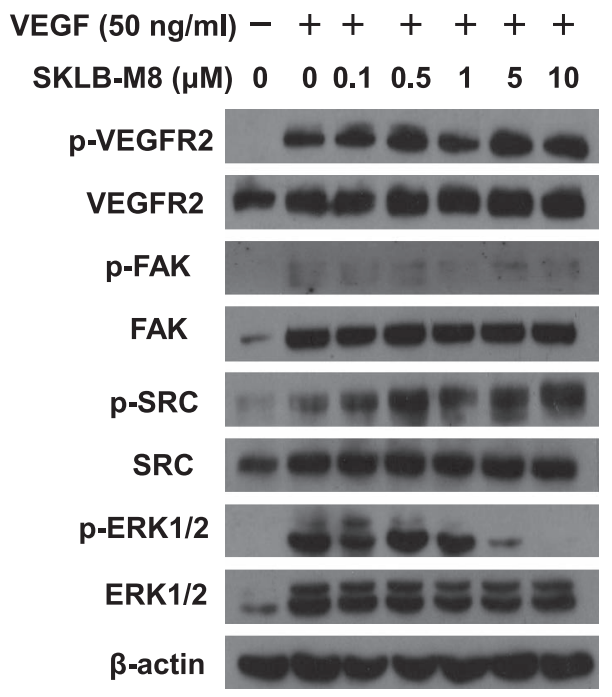

Fig. 4. SKLB-M8 inhibited migration, invasion, and tube formation of HUVECs. A: SKLB-M8 inhibited HUVEC migration in wound healing assay. The left image represents wounded cells before treatment. B: SKLB-M8 inhibited HUVECs invasion in the Transwell assay. After treatment with vehicle or SKLB-M8 for $24 \mathrm{~h}$, the HUVECs that invaded through the membrane were stained and quantified. C: SKLB-M8 inhibited tube formation of HUVECs. After treated with vehicle or SKLB-M8 for 6 h, vascular tube structure was quantified by manual counting. The percentage of inhibition was expressed using vehicle-treated cells at 100\%. D: Western blotting analysis of HUVECs following SKLB-M8 treatment. SKLB-M8 suppressed phosphorylation of ERK1/2, with no significant effect on the activities of VEGFR2, FAK, and Src. Columns, mean; bars, S.D. $(\mathrm{n}=3) . * P<0.05$, $* * P<0.01$, and $* * * P<0.001$, significantly different compared with the control by the $t$-test.

only), no signification differences in weights were found among the 3 groups in the two mouse models. In addition, toxic pathologic changes in heart, liver, spleen, lungs, and kidneys were not observed by microscopic examination (Supplementary Fig. 1B). The results revealed that SKLB-M8 was an effective antitumor compound with less toxicity.

\section{Discussion}

Previous reports showed that P53 target genes involved in cell cycle regulation and apoptosis are aberrantly expressed in melanoma, and this aberrant functional activity may affect the proliferation of melanoma (26, 27). In our study, SKLB-M8 significantly inhibited the proliferation and blocked the cell cycle process of melanoma cell lines by down-regulating cdc2 and increasing p53 expression. Western blotting and immunochemistry verified that SKLB-M8 induced apoptosis of melanoma with activation of caspase-3 (Figs. 3B, 6F).

Inhibition of AKT can enhance p53-mediated apoptosis and promote Bax conformational change, which leads to disruption of the mitochondrial membrane potential and caspase- 3 activation $(28-31)$. A cytostatic cellular response caused by the inhibition of mTOR greatly enhanced the proapoptotic effect of cytotoxic agents through different mechanisms, including down-regulating antiapoptotic proteins of the Bcl-2 family $(32,33)$. As shown in Fig. 3C, SKLB-M8 inhibited the expression of AKT and p-mTOR, which revealed that SKLB-M8 might induce apoptosis of melanoma cells by the AKT/ mTOR pathway following p53 up-regulation.

Anti-angiogenesis was another characteristic of SKLB-M8. The cellular signaling pathways mediated by VEGFR2 are relevant to tumor growth and angiogenesis (34). Its downstream proteins, Src, FAK, and ERK are substantially involved in VEGF-induced angiogenesis as well as regulation of cell motility and vascular permeability $(35-37)$. Our study indicated that SKLBM8 seemed not to inhibit the activation of VEGFR2, FAK, and Src, but obviously suppressed the expression of p-ERK under the employed concentrations $(5-10 \mu \mathrm{M})$, which were near the $\mathrm{IC}_{50}(6.45 \mu \mathrm{M})($ Fig. 4D).

ERK kinases contribute to microtubule stability, which is required for cellular remodeling that occurs during proliferation, migration, and invasion of normal cells 

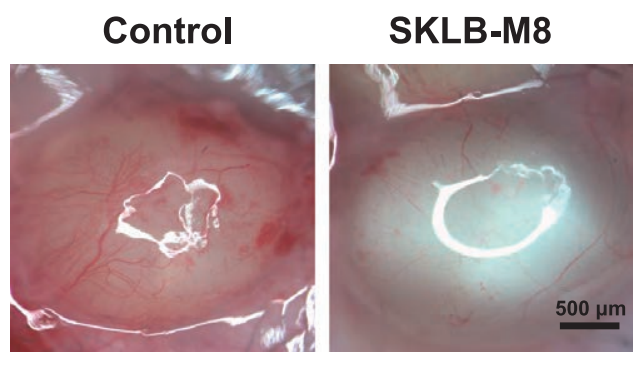

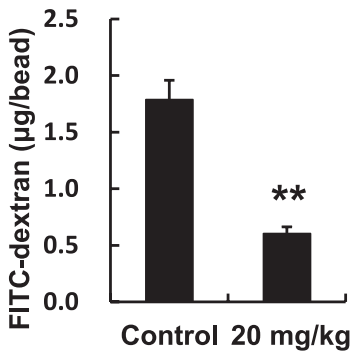

Fig. 5. SKLB-M8 inhibited tumor angiogenesis in vivo. Alginate Beads were surgically removed at the end of the experiment, and FITC-dextran was quantified. FITCdextran uptake of beads from SKLB-M8-treated mice showed a significant decrease compared with the vehicle control group $\left(\mathrm{n}=6\right.$; $t$-test, $\left.{ }^{* *} P<0.01\right)$.
A

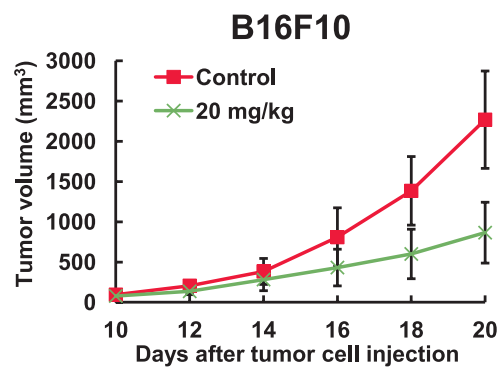

C

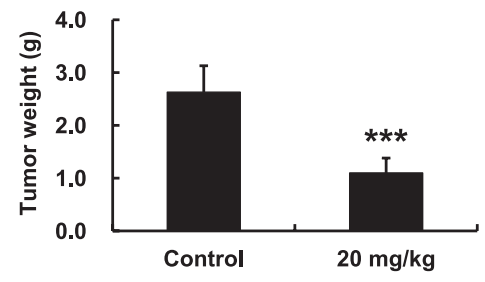

B

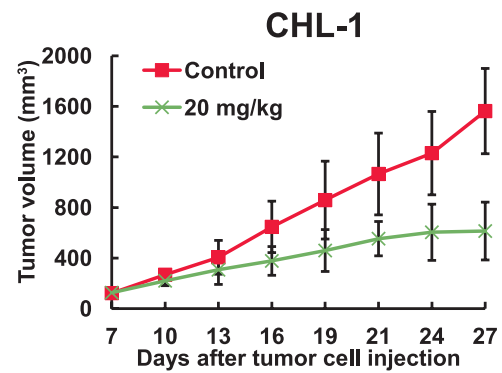

D

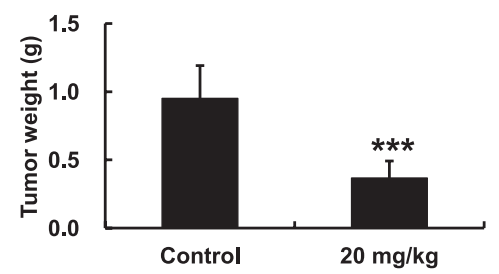

E
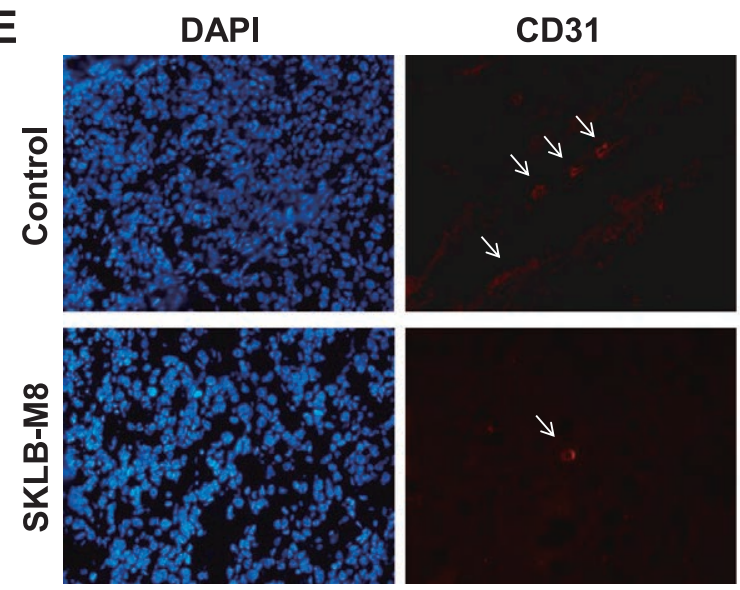

$\mathbf{F}$

Control

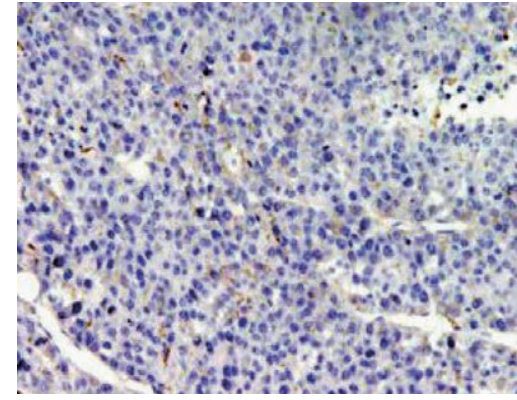

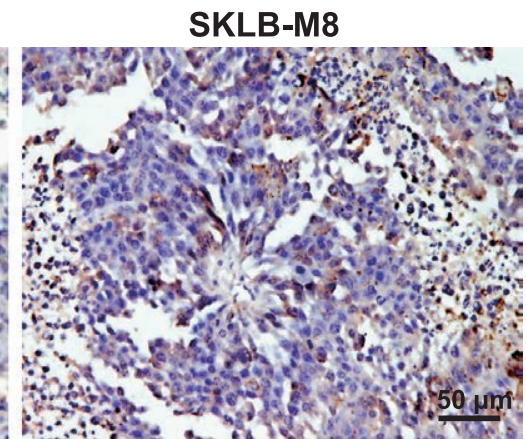

Fig. 6. SKLB-M8 inhibited tumor growth in vivo. $\mathrm{A}$ and $\mathrm{B}$ : Tumor volume-time curve of B16F10 or CHL-1 model. The $20 \mathrm{mg} / \mathrm{kg}$ group was treated orally with $200 \mu \mathrm{l}$ of physiological saline with SKLB-M8 daily and the control group received orally physiological saline alone $(\mathrm{n}=6$ per group). The treatment with SKLB-M8 resulted in significant inhibition of tumor growth vs. control. C and D: The bar charts of tumor weight of B16F10 or CHL-1 model. The average weight of B16F10 and CHL-1 tumors from the control group were compared with $2.63 \pm 0.50$ or $0.95 \pm$ $0.12 \mathrm{~g}$, respectively, whereas that in the $20 \mathrm{mg} / \mathrm{kg}$ SKLB-M8-treated groups were $1.10 \pm 0.28$ or $0.37 \pm 0.12$ g, respectively. $* * * P<0.001$, significantly different compared with the control by the $t$-test. E: SKLB-M8 significantly inhibited tumor vessels. Frozen sections of B16F10 tumor tissue were tested by immunofluorescence analysis with anti-CD31 antibody. F: Apoptosis was measured on paraffin-embedded B16F10 tumor sections by immunohistochemistry. The apoptotic index was calculated by dividing the number of active caspase-3-expressing cells by the total number of cells. 
and cancer cells $(38-40)$. Activation of ERK by the ras pathway coincided with a decrease in the stability of microtubules, mediating enhanced proliferation $(39,41)$. Thus, we inferred that SKLB-M8 might inhibit HUVECs proliferation by suppressing the activation of ERK. As shown in Fig. 4, SKLB-M8 obviously inhibited HUVECs migration, invasion, and tube formation with much lower concentrations than $\mathrm{IC}_{50}$ and there must be other pathways affecting the results. Our studies have revealed that SKLB-M8 is a novel tubulin inhibitor. Useful tools have been provided by microtubule-binding drugs and vascular-disrupting agents to demonstrate that tubulin polymerization and stabilization are required for formation and maintenance of angiogenic structures, respectively (22). Hence, it seemed that SKLB-M8 inhibited HUVEC migration, invasion, and tube formation through disrupting microtubule stability, and ERK was one of the important mediators. The functional role for microtubules in angiogenesis and the mechanism should be investigated in future investigations.

In animal experiments, SKLB-M8 significantly inhibited tumor growth of B16F10 and CHL-1, and we did not find that SKLB-M8 affected the body weight of the mice in both models and did not cause any toxic pathologic changes in normal tissues (Supplementary Fig. 1). However, the poor solubility of SKLB-M8 limited the effect of treatment. Our following research will focus on the suitable pharmaceutics formation. In conclusion, our study revealed that SKLB-M8, a novel agent, inhibited the growth of melanoma cells both in vitro and in vivo. In vitro, SKLB-M8 inhibited the expression of cdc2 and up-regulated p53 to induce G2/M arrest in A2058 and CHL-1 cells and induced cell apoptosis through down-regulating AKT and p-mTOR in B16F10, A2058, and CHL-1 cells. Further, SKLB-M8 suppressed tumor angiogenesis by decreasing ERK phosphorylation. In B16F10 and CHL-1 models, SKLB-M8 showed significant antitumor activity with less toxicity. Thus, SKLBM8 might be a potential anti-melanoma drug candidate.

\section{Acknowledgments}

The authors greatly appreciate the financial support from National Key Programs of China during the 12th Five-Year Plan Period (2012ZX09103101-009), National Natural Science Foundation of China (81373283).

\section{Conflicts of Interest}

The authors indicated no potential conflicts of interest.

\section{References}

1 Jerant AF, Johnson JT, Sheridan CD, Caffrey TJ. Early detection and treatment of skin cancer. Am Fam Physician. 2000;62: 357-368.

2 Koehn FE, Carter GT. The evolving role of natural products in drug discovery. Nat Rev Drug Discov. 2005;4:206-220.

3 Newman DJ, Cragg GM. Natural products as sources of new drugs over the last 25 years. J Nat Prod. 2007;70:461-477.

4 Schwarzenberg K, Vollmar AM. Targeting apoptosis pathways by natural compounds in cancer: marine compounds as lead structures and chemical tools for cancer therapy. Cancer Lett. 2013;332:295-303.

5 Rajeev S, Samant RS, Shevde LA. Recent advances in antiangiogenic therapy of cancer. Oncotarget. 2011;2:122-134.

6 Kastan MB, Canman CE, Leonard CJ. P53, cell cycle control and apoptosis: implications for cancer. Cancer Metastasis Rev. 1995;14:3-15.

7 Levine AJ. p53, the cellular gatekeeper for growth and division. Cell. 1997;8:323-331.

8 Taylor WR, Stark GR. Regulation of the G2/M transition by 553 . Oncogene. 2001;20:1803-1815.

9 Testa JR, Bellacosa A. AKT plays a central role in tumorigenesis. PNAS. 2001;98:10983-10985.

10 Mi C, Ma J, Shi H, Li J, Wang F, Lee JJ, et al. 4',6-Dihydroxy4-methoxyisoaurone inhibits the HIF-1 $\alpha$ pathway through inhibition of $\mathrm{Akt} / \mathrm{mTOR} / \mathrm{p} 70 \mathrm{~S} 6 \mathrm{~K} / 4 \mathrm{E}-\mathrm{BP} 1$ phosphorylation. J Pharmacol Sci. 2014;125:193-201.

11 Wu WS, Ye HY, Wan L, Han XL, Wang GC, Hu J, et al. Millepachine, a novel chalcone, induces G2/M arrest by inhibiting CDK1 activity and causing apoptosis via ROSmitochondrial apoptotic pathway in human hepatocarcinoma cells in vitro and in vivo. Carcinogenesis. 2013;34:1636-1643.

12 Jaffe ER, Nachman RL, Becker CG, Minick CR. Culture of human endothelial cells derived from umbilical veins. Identification by morphologic and immunologic criteria. J Clin Invest. 1973;52:2745.

13 Timke C, Zieher H, Roth A, Hauser K, Lipson KE, Weber KJ, et al. Combination of vascular endothelial growth factor receptor/platelet-derived growth factor receptor inhibition markedly improves radiation tumor therapy. Clin Cancer Res. 2008;14: 2210-2219.

14 Pang XF, Yi ZF, Zhang XL, Sung B, Qu WJ, Lian XY, et al. Acetyl-11-Keto- $\beta$-boswellic acid inhibits prostate tumor growth by suppressing vascular endothelial growth factor receptor 2-mediated angiogenesis. Cancer Res. 2009;69:5893-5900.

15 Hoffmann J, Schirner M, Menrad A, Schneider MR. A highly sensitive model for quantification of in vivo tumor angiogenesis induced by alginate-encapsulated tumor cells. Cancer Res. 1997;57:3847-3851.

16 Shrimali RK, Yu ZY, Theoret MR. Antiangiogenic agents can increase lymphocyte infiltration into tumor and enhance the effectiveness of adoptive immunotherapy of cancer. Cancer Res. 2010;70:6171-6180.

17 Gautier J, Minshull J, Lohka M, Glotze M, Hunt T, Maller JL. Cyclin is a component of maturation-promoting factor from Xenopus. Cell. 1990;60:487-494.

18 Utz P, Anderson P. Life and death decisions: regulation of apoptosis by proteolysis of signaling molecules. Cell Death Differ. 2000;7:589-602. 
19 Uchida M, Hanai S, Uematsu N, Sawamoto K, Okano H, Miwa $\mathrm{M}$, et al. Genetic and functional analysis of PARP, a DNA strand break-binding enzyme. Mutat Res. 2001;77:89-96.

20 Cardone MH, Roy H, Stennicke HR, Salvesen GS, Franke TF, Stanbridge E, et al. Regulation of cell death protease caspase-9 by phosphorylation. Science. 1998;282:1318-1321.

21 Dormond O, Madsen JC, David. The effects of mTOR-Akt Interactions on anti-apoptotic signaling in vascular endothelial cells. J Biol Chem. 2007;282:23679-23686.

22 Bayless KJ, Johnson GA. Role of the cytoskeleton in formation and maintenance of angiogenic sprouts. Journal of vascular research. 2011;48:369-385.

23 Ferrara N, Kerbel RS. Angiogenesis as a therapeutic target. Nature. 2005;438:967-974.

24 Zhang S, Cao Z, Tian H, Shen G, Ma Y, Xie H, et al. SKLB1002, a novel potent inhibitor of VEGF receptor 2 signaling, inhibits angiogenesis and tumor growth in vivo. Clin Cancer Res. 2011; 17:4439-4450.

25 Chen X, Ji P, Yang HW, Yang LL, Zhou S, Zhong L, et al. SC-535, a novel oral multikinase inhibitor, showed potent antitumor activity in human melanoma models. Cell Physiol Biochem. 2013;32:138-153.

26 Houben R, Hesbacher S, Schmid CP, Kauczok CS, Flohr U, Haferkamp S, et al. High-level expression of wild-type p53 in melanoma cells is frequently associated with inactivity in $\mathrm{p} 53$ reporter gene assays. PLoS ONE. 2011;6:e22096.

27 Avery-Kiejda KA, Bowden NA, Croft AJ, Scurr LL, Kairupan $\mathrm{CF}$, Ashton KA, et al. P53 in human melanoma fails to regulate target genes associated with apoptosis and the cell cycle and may contribute to proliferation. BMC Cancer. 2011;11:203.

28 Kojima K, Shimanuki M, Shikami M, Samudio IJ, Ruvolo V, Corn P, et al. The dual PI3 kinase/mTOR inhibitor PI-103 prevents p53 induction by Mdm2 inhibition but enhances p53-mediated mitochondrial apoptosis in p53 wild-type AML. Leukemia. 2008;22:1728-1736.

29 Parcellier A, Tintignac LA, Zhuravleva E, Hemmings BA. PKB and the mitochondria: AKTing on apoptosis. Cellular Signalling. 2008;20:21-30.

30 Yamaguchi H, Wang HG. The protein kinase PKB/Akt regulates cell survival and apoptosis by inhibiting Bax conformational change. Oncogene. 2001;20:7779-7786.
31 Arokium H, Ouerfelli H, Velours G, Camougrand N, Vallette FM, Manon S. Substitutions of potentially phosphorylatable serine residues of Bax reveal how they may regulate its interaction with mitochondria. J Biol Chem. 2007;282:35104-35112.

32 Tirado OM, Mateo-Lozano S, Notario V. Rapamycin induces apoptosis of JN-DSRCT-1 cells by increasing the Bax : Bcl-xL ratio through concurrent mechanisms dependent and independent of its mTOR inhibitory activity. Oncogene. 2005;24:3348-3357.

33 Vega F, Medeiros LJ, Leventaki V. Activation of mammalian target of rapamycin signaling pathway contributes to tumor cell survival in anaplastic lymphoma kinase-positive anaplastic large cell lymphoma. Cancer Res. 2006;66:6589-6597.

34 Nakatsu MN, Sainson RCA, Pérez-del-Pulgar S, Aoto JN, Aitkenhead M, Taylor KL, et al. VEGF121 and VEGF165 regulate blood vessel diameter through vascular endothelial growth factor receptor 2 in an in vitro angiogenesis model. Lab Invest. 2003;83:1873-1885.

35 Mitra SK, Schlaepfer DD. Integrin-regulated FAK-Src signaling in normal and cancer cells. Curr Opin Cell Biol. 2006;18: 516-523.

36 Chou MT, Wang J, Fujita DJ. Src kinase becomes preferentially associated with the VEGFR, KDR/Flk-1, following VEGF stimulation of vascular endothelial cells. BMC Biochemistry. 2002; 3:32.

37 Dai J, Peng L, Fan K, Wang H, Wei R, Ji G, et al. Osteopontin induces angiogenesis through activation of PI3K/AKT and ERK1/2 in endothelial cells. Oncogene. 2009;28:3412-3422.

38 Elbaum M, Chausovsky A, Levy ET, Shtutman M, Bershadsky AD. Microtubule involvement in regulating cell contractility and adhesion-dependent signalling: a possible mechanism for polarization of cell motility. Biochemical Society symposium. 1999;65:147-172.

39 Jordan MA, Wilson L. Microtubules and actin filaments: dynamic targets for cancer chemotherapy. Curr Opin Cell Biol. 1998;10:123-130.

40 Li Y, Lu X, Qi H, Li X, Xiao X, Gao J. Ursolic acid induces apoptosis through mitochondrial intrinsic pathway and suppression of ERK1/2 MAPK in HeLa cells. J Pharmacol Sci. 2014;125:202-210.

41 Harrison RE, Turley EA. Active Erk regulates microtubule stability in H-ras-transformed cells. Neoplasia. 2001;3:385-394. 